Chin-Te Jung ${ }^{1}$, Chih-Hong Sun ${ }^{2}$, Min-Fang Lien ${ }^{3}$, Chih-Shyang Chang ${ }^{4}$, Wei-Jen Chung ${ }^{5}$, Hong-Yang Lin ${ }^{6}$, Ping-Ying Tsai ${ }^{7}$

${ }^{1}$ Ph.D., Department of Geography, National Taiwan University, Taipei city, Taiwan, No. 1, Sec. 4, Roosevelt Road, 106, Tel: +886-2-33665829, chinte.jung@gmail.com

2 Professor, Department of Geography, National Taiwan University, Taipei city,

Taiwan, No. 1, Sec. 4, Roosevelt Road, 106, Tel: +886-2-33665829, chsun2145@gmail.com

* Corresponding author

${ }^{3}$ Director of Technologies Department, Taiwan GIS Center, Taipei city, Taiwan, 6F, No. 6, Sec. 1, Roosevelt Road, 106, Tel: +886-2-23931122, collmf@tgic.org.tw

${ }^{4}$ Professor, Department of Information Management, Tungnan University, New Taipei city, Taiwan, No. 152, Sec. 3, Beishen Road, 222, Tel: +886-2-86625945, y47106@gmail.com

${ }^{5}$ Engineer, Taiwan GIS Center, Taipei city, Taiwan, 6F, No. 6, Sec. 1, Roosevelt Road, 106, Tel: +886-2-23931122, william@tgic.org.tw

${ }^{6}$ Engineer, Taiwan GIS Center, Taipei city, Taiwan, 6F, No. 6, Sec. 1, Roosevelt Road, 106, Tel: +886-2-23931122, linhy@tgic.org.tw

${ }^{7}$ Engineer, Taiwan GIS Center, Taipei city, Taiwan, 6F, No. 6, Sec. 1, Roosevelt Road, 106, Tel: +886-2-23931122, bytsai0808@tgic.org.tw

\title{
MAKOCI:
}

\section{A WEB PORTAL FOR INTEGRATING AND SHARING GEOGRAPHIC INFORMATION SERVICES}

\begin{abstract}
The lack of integration and communication of various geographic information services (Gl services) has resulted in many duplication collection of earth observation data, and challenges of semantic interoperability. This paper proposes an ontology-based multi-agents platform, called MAKOCI (multi-agent knowledge oriented cyberinfrastructure), which acts as Gl service one stop to manage, publish, share, and discover Gl services semantically. By ontologies, formal meanings of concepts are defined to annotate and discover $\mathrm{Gl}$ services on a conceptual level for semantic interoperability. With the assistance of multi-agents, the processes in MAKOCI can be divided into various modules and be communicated based on the same semantics in ontologies. A prototype was implemented to test MAKOCI. Finally, we conclude the advantages and disadvantages of MAKOCI and point out several future works.
\end{abstract}

KEYWORDS: Geographic Information Service, Ontologies, Multi-agent System, Cyberinfrastructure

\section{INTRODUCTION}

The explosive growth of the Internet in recent years has led to significant advances in the use of Web services, instead of conventional software tools, for processing data and exchanging information [Kvaløy et al., 2005]. In earth observations, observed data (e.g., satellite images or phenomenon measured by in-situ sensors) can be encoded into Web services for sharing and communication. The Open Geospatial Consortium (OGC) recently has built a unique and revolutionary framework of open standards, called sensor Web enablement (SWE), for exploiting Webconnected sensors and sensor systems of all types: flood gauges, air pollution monitors, stress gauges on bridges, mobile 
heart monitors, Webcams, satellite-borne earth imaging devices and countless other sensors and sensor systems via Web services [Jaeger et al., 2005]. The observed data by these sensors are often accompanied with geographical information, such as place names or coordinates, and can be considered as one type of geographic information services (Gl services). With the assistance of OGC, there are more standard formats for Gl services, such as geography markup language (GML), Web mapping service (WMS), Web feature service (WFS), and Web processing service (WPS), have dominated the Web to perform geospatial data acquisition and processes as well as information integration and exchange [Peng and Tsou, 2003]. Nevertheless, while sharing and integrating heterogeneous GI services, a central platform for search required GI services and semantically interoperate these Gl services [Egenhofer, 2002; Kuhn, 2003] appear more critical.

Semantic interoperability of geospatial data has been recognized as the main hamper for integrating $\mathrm{Gl}$ services [Fonseca and Sheth, 2002]. It is because that different terminology may be used to describe the same phenomenon by different domains and it lacks a common and shared understanding across the domains to interoperate and communicate [Kuhn, 2003]. For example, one may use AirTemperature to describe an observation of air temperature in a Gl service, whereas the other one may use ATemp to express the same observation. It will be difficult to integrate those two Gl services without a shared understanding on the terminologies.

In the other hand, a central platform, such as Geospatial One-Stop', is lack for Gl services to be registered, discovered, and managed. Data providers can easily use GIS-Server software, such as GeoServer ${ }^{2}$, to publish their observation data into standard Web services over the Web. However, it may challenges for other users to use the Web services if they do not know where they are. In addition,

\footnotetext{
http://geo.data.gov

2 http://geoserver.org/display/GEOS/Welcome
}

semantic heterogeneity described above hampered among users as well, especially when they use different keywords to search Gl services in the platform.

Therefore, this study aims to develop an ontology-enabled multi-agent platform, whichfacilitates ontologies to define common understandings for semantic interoperability, and uses multi agents to assist Web service providers and consumers to register and discovery Gl services in the platform. The platform is called multi-agent knowledge oriented cyberinfrastructure (MAKOCl). We divide GI services into two categories: dataaccessing services (Web services that provide GIS data) and geoprocessing services (Web services that analyze GIS data) [Lutz et al., 2007]. The metadata of each Gl service is aimed to be registered into $\mathrm{MAKOCl}$ and use defined formal meanings in ontologies to annotate the registered metadata for semantic interoperability. The registered metadata is encoded in the resource description framework (RDF) [Klyne and Carroll, 2004] so that Web service consumers can search appropriate Gl services not only by browsing categories or using keywords but also by using SPARQL, a query language for RDF [Prud'Hommeaux and Seaborne, 2008], on the semantic level.

This paper is organized as follows. Section 2 briefly introduces the background about ontologies, RDF, and multi agents. Section 3 presents the proposed framework of MAKOCI. Section 4 shows an implemented prototype. Section 5 concludes the advantages and disadvantages of the platform and points out some future works.

\section{BACKGROUND}

\section{Ontologies}

In information science, an ontology is defined as "an explicit specification of a conceptualization" [Gruber, 1993], where a conceptualization is a way of "thinking about a domain"[Uschold, 1998] whereas the formal meanings of both the types of concepts and their relationships in a domain are 
delineated in a machine-readable language by the explicit specifications for semantic interoperability and knowledge inference [Benjamins et al., 1998]. By Ontologies, shared and common understandings (i.e., semantics) can be communicated among people and machines [Crubézy and Musen, 2003]. The formal meanings in an ontology can be incorporated into data integration processing [Peachavanish and Karimi, 2007], search algorithms [Bernard et al., 2003; Purves et al., 2007; Stock, 2008], and analytical methods [Di et al., 2006; Lutz et al., 2007].

The Web ontology language (OWL) is the standard language of ontologies and is recommended by W3C [McGuinness and Van Harmelen, 2004]. Basic elements of OWL include individuals, classes, and properties (i.e., relationships) [Knublauch et al., 2004]. Individuals represent real instances in a domain, whereas classes represent concepts that can be seen as collections of individuals of the same type. Properties are binary and directional links that connect individuals from one class (called the domain of the property) to another (called the range of the property). Properties can be classified on the basis of this range into object and datatype properties [McGuinness and Van Harmelen, 2004]. Object properties link individuals, whereas datatype properties link an individual to an XML schema datatype (e.g., an integer or string) [Knublauch et al., 2004].

A solution to the semantic interoperability for $\mathrm{Gl}$ services is to develop ontologies in geospatial domains to identify semantic distinctions and relations between geospatial concepts based on ontological specifications, which is a key factor suggested by Egenhofer [2002] for a geospatial semantic Web. Geospatial ontologies can be the foundation for achieving geospatial semantic interoperability [Bishr, 1998; Harvey et al., 1999; Egenhofer, 2002; Fonseca and Sheth, 2002; Kuhn, 2003; Lemmens, 2006], improving geospatial data searches [Purves et al., 2007], and discovering Gl services in an SDI [Bernard et al., 2003; Lutz, 2005, 2006; Zhang and Tsou, 2009].

\section{$R D F$}

The RDF is one of standard frameworks for semantic Web recommended by World Wide Web Consortium (W3C) [Klyne and Carroll, 2004]. The RDF aims to represent information in the Web by formal semantics, which can be readable and accessible by machines to query and infer implicit information. The RDF encodes the information in of subject, predicate, and object triplets [Klyne and Carroll, 2004; Bizer et al., 2009]. The subject and object of a triple are uniform resource identifiers (URIs) to identify resources (e.g., concepts and individuals in ontologies) or XML schema datatype, whereas the predicate specifies the relationships between the subject and object, and is also represented by URIs [Bizer et al., 2009]. URI is similar to a uniform resource locator (URL), which use the http://scheme to describe an address of a website. However, URI provide a more generic means to identify resources over the Web by the HTTP protocol [Klyne and Carroll, 2004].

For example, an RDF triple can state that a Gl service, Taiwan_River, has the polyline geometry, as shown in Fig. 1. The subject and object of the triple are Taiwan_River and the polyline geometry which is related by the predicate, hasGeometry. URIs are used to identify each resource in the RDF triple, such as http://www.tgic.org.tw/GISData. owl\#Taiwan_River for the subject, http:// www.tgic.org.tw/GIS.owl\#hasGeometry for the predicate, and http://WwW.tgic.org.tw/ Geometry.owl\# Polyline for the object.

Moreover, machines not only can understand the relationship between the subject and object in an RDF triple but also can access further information via URIs. For instance, in Fig. 1, machines can use the URI of the subject, http://www.tgic.org.tw/GISData. owl\#Taiwan_River, which is referred to GIS Data ontology (i.e., GISData.owl) to access more specifications about the subject.

\section{Multi-agent system}

Agents are computer programs capable of autonomous behavior which can sense its environment, communicate with other 


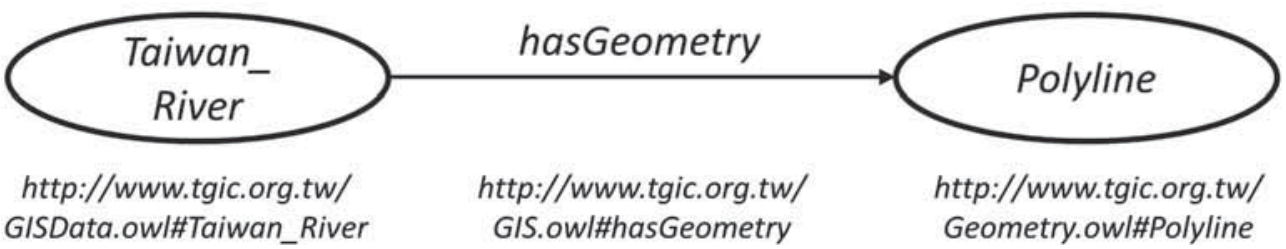

Fig. 1. An RDF triple states that a GI service, Taiwan_River, has the polyline geometry

agents, and possess rational behavior to react [Wooldridge and Jennings, 1995; Wooldridge, 1999]. Multi-agent system are referred to a system that support interactions from multiple agents with an infrastructure [Moodley and Simonis, 2006]. For the purpose of agents, two types of agents can be classified: 1) simulating the perceived or measure behaviors of people response to external stimuli using computational models of rational behaviors, and 2) assisting people to collect information and making decision in hardware and software environments [Sengupta and Sieber, 2007]. In this study, agents are acted the latter one in a software environment to detect and analyze users' behaviors, and automatically collect related information for the users.

However, agents are only computer programs and cannot automatically handle semantic heterogeneous of information without rational behaviors or rules. Without ontologies supports, agents are challenged

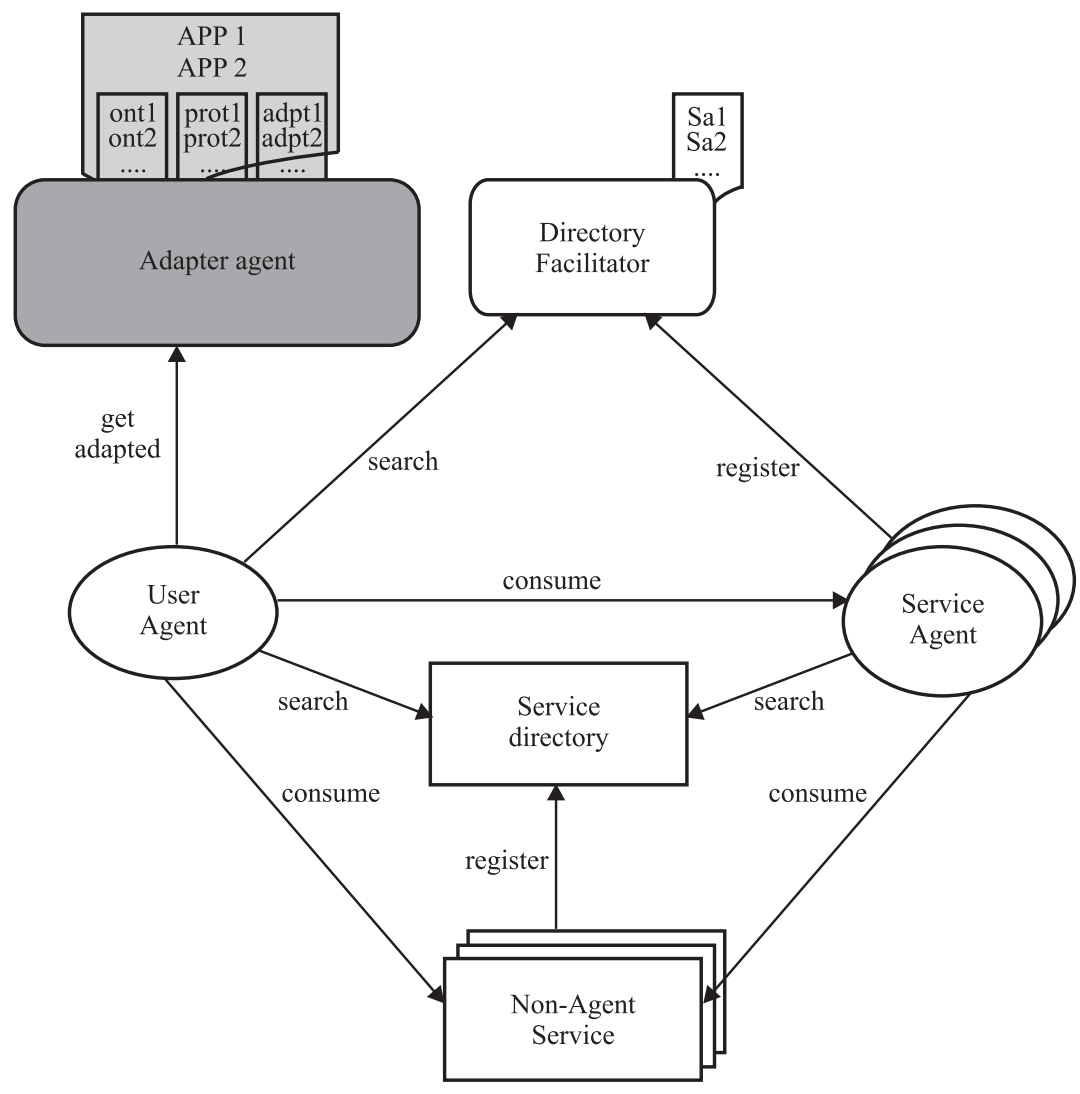

Fig. 2. MASII architecture [Moodley and Kinyua, 2006] 
to exchange information based on a common and shared understanding [Hadzic et al., 2009]. For instances, Rauble [Rauble, 2001] has used ontologies to model common knowledge for the communication of agents while perceiving, cognizing, and acting for the way finding; and Moodley and Simonis [Moodley and Simonis, 2006] proposed a platform, which is based on the multi-agent infrastructure for distributed Internet applications (MASII) by Moodley [Moodley and Kinyua, 2006], aimed to integrate heterogeneous sensor data with ontologies that facilitate developing applications for multiple domains.

The MASII architecture contains several agents to communicate information under the shared semantic in ontologies, as shown in Fig. 2. The agents includes 1) user agent which represent an end-user to communicate with other agents 2) service agent is a service provider which can be searched in the directory facilitator and be consumed by the user agent, 3) nonagent service which expresses conventional web services to be consumed by the user agent and the service agent, 4) adapter agent which maintains common knowledge and semantic in ontologies for semantic interoperability among agents.

Nevertheless, how to register and semantic infer sensor web services is not presented in [Moodley and Kinyua, 2006; Moodley and Simonis, 2006]. Thus, in this paper, we based on the MASII architecture and proposed an ontology-enabled multi-agents platform with a practical application for complement. Please refer to Section 3 for more detail about the proposed platform.

\section{AN ONTOLOGY-ENABLED MULTI-AGENTS PLATFORM (MAKOCI)}

\section{The framework of MAKOCI}

$\mathrm{MAKOCl}$ aims to develop a platform for geospatial cyberinfrastructure, an Webbased environment for the integration of geospatial knowledge, data, and technologies [Yang et al., 2010]. MAKOCl adopts multi-agents and ontologies to entail Gl services (as geospatial data) with geospatial knowledge for the collaboration of domain experts to contribute their domain knowledge, Web service providers to publish and semantically annotate their Gl services, and Web service consumers to semantically search and use their required Gl services. Ontologies are used for semantic interoperability and knowledge sharing while registering or searching Gl services. And multi-agents can be intelligent to assist users to automatically discover Gl services in ontologies in response of users' queries, and can be autonomously communicated each agents for exchanging information. The MAKOCI framework contains the following two levels, as shown in Fig. 3:

(1) The application level includes (a) ONTOCAT (ontology catalog) application, which provides a registry for Web service providers and consumers to register and discover Gl services assisted by ontologies and multi-agents; (b) ONTOEDIT (ontologies editor), which offers an environment for domain experts to construct domain knowledge into ontologies; and (c) iSDSS (intelligent spatial decision support system), which supplies an integrated application for Web service consumers to use discovered Gl services in ONTOCAT.

(2) The agent level involves Safeguard agent, User agent, Ontology agent, Service agent, and a Directory Facilitator. The Directory Facilities is an agent's registry where agents register their capabilities so that agents can be discoverable [Moodley and Simonis, 2006]. While users log into the applications in MAKOCl, a Safeguard will be initialized to examine the authorities of the users and search an available User agent in the Directory Facilitator. Once a available User agent is found, the User agent will serve the users by analyzing users' activities and searching available Ontology agents (for the references of formal concepts in ontologies) and Service agents (to translate requests into service-specific standards and call the services). In addition, the users' activities will be recorded and stored for the analysis 


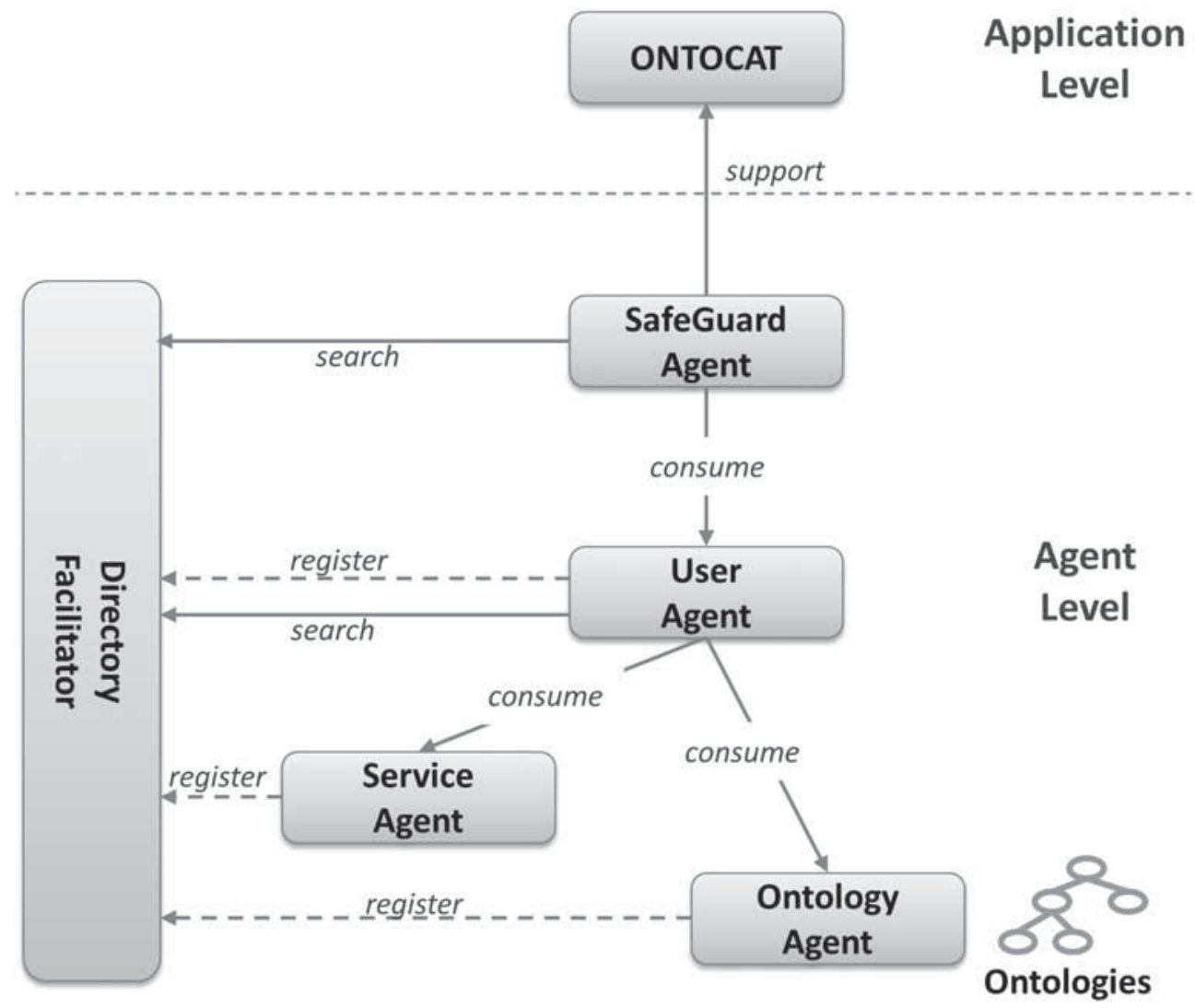

Fig. 3. The framework of MAKOCI

of users' behaviors and for the automatic recommendations of relevant $\mathrm{Gl}$ services.

\section{Developed Ontologies}

The communication among these agents is based on the ontologies to control vocabularies and refer to the formal meaning of concepts and their relationships for semantic interoperability. Ontologies are located at the center of the framework and are developed in the 7 related geospatial domains, which are based on the suggestions of Kolas [2005].

\section{GIS ontology}

The GIS ontology delineates the relationships and definitions of geospatial features, which are abstractions of real-world phenomena with types of geometries (e.g., points, polylines, and polygons) and attributes [Moodley and Kinyua, 2006]. Three OGC specifications can be followed to develop the GIS ontology.

The "OGC Abstract Specification Topic 5: Feature" stipulates that a geospatial feature should contain a geometry (e.g., point, polyline, or polygon) with a spatial reference system and attributes [Moodley and Kinyua, 2006]. For example, in Fig. 4, Feature, Attribute, Geometry, Spatial Reference System, and Feature Collection (i.e., a set of features with the same attributes, geometries, and spatial references) are created as classes in the GIS ontology, whereas hasGeometry, hasAttribute, and hasSpatialReference are set as object properties to describe the relationships between these classes.

The "OGC Abstract Specification Topic 2: Spatial Referencing by Coordinates" extends the classes under the Spatial Reference 


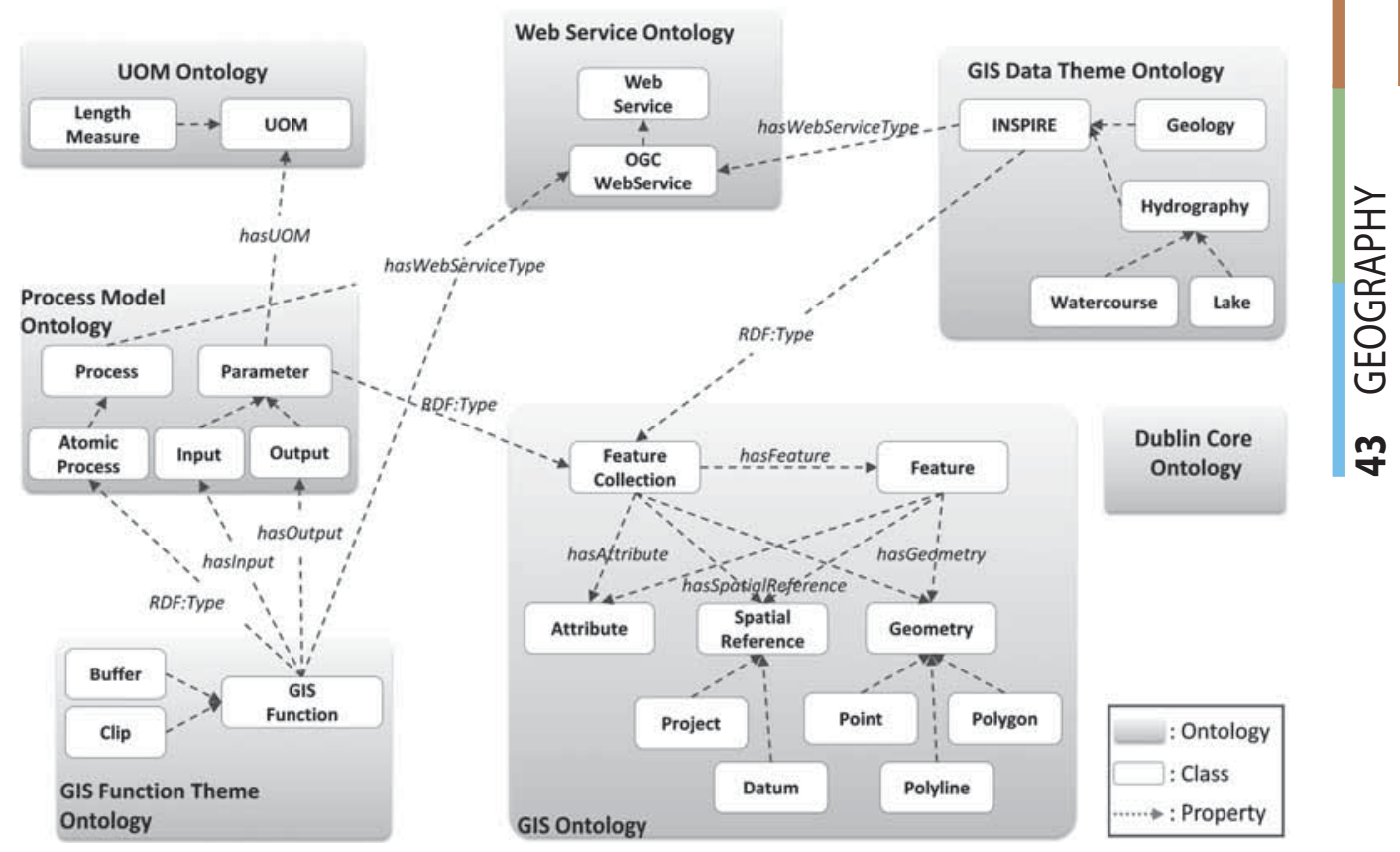

Fig. 4. The developed ontologies

Systems, such as for Datum and Project in Fig. 4 [Babitski et al., 2009].

The "OGC Simple Feature Access Part 1: Common Architecture" provides categorization of common geospatial geometries, which can be expanded as classes under the OGCGeometry class [OGC, 2006a]. However, we here consider only vector-type geometries such as points, polylines, and polygons, leaving raster geometry for future work.

\section{Web Service ontology}

The Web Service ontology specified standards for $\mathrm{Gl}$ services. Here, we focus only on the OGC Web Service standards, e.g., WFS [OGC, 2005], WMS [OGC, 2006b], and WPS [Goodwin and Russomanno, 2006], which are created as individuals belonging to the OGC Web Service class.

\section{GIS Data Theme ontology}

The GIS Data Theme ontology manages the classification of GIS data. This study follows the classification from Infrastructure for Spatial Information in the European Community (INSPIRE) to establish this ontology. The classification contains 34 spatial data themes, which are grouped in 3 annexes. For each data theme, we can further customize spatial data components and spatial data sets. For example, Lake and Watercourse can be added as subcategories of the Hydrography.

\section{GIS Function Theme ontology}

The GIS Function Theme ontology describes the 6 main categories and 20 universal operations proposed by Albrecht [1998] to structure GIS function classification. Universal operations are GIS operations, which are independent of data structures, and are classified from 144 GIS functions into 20 categories (e.g., spatial search, thematic search, and reclassification) [Albrecht, 1998]. Under the classification, other structure-independent GIS functions can be created as classes to customize the GIS Function Theme ontology. For example, in Fig. 5, Overlay is a universal GIS operation categorized by Locational Analysis. Under the Overlay operation, we can add Clip as a new class to extend the classification. Alternatively, Measurement is a universal GIS operation classified under the Measurements category, and Sum can be added as a structure-independent GIS function under the Measurement operation. 
class and has to satisfy the restrictions on the FeatureCollection class, as shown in Fig. 5.

Moreover, the preconditions and results of a geoprocessing service can be annotated by the hasPrecondition and hasResult properties using specific expressions [Janowicz et al., 2010]. A precondition is a logical formula stating that a condition should be satisfied before execution, whereas a result is a logical formula expressing what will be true upon successful execution. For example, the distance individual must have values before execution (precondition), whereas polygon geometry may be generated after successful execution (result). However, for simplicity, we focus only on the inputs and outputs of a geoprocessing service in this study. Further annotations of preconditions and results are listed as part of our future work.

\section{Units of Measure ontology}

The Units of Measure ontology is adopted from the suggested upper merge ontology (SUMO), an upper-level ontology providing definitions of general-purpose terms [Niles and Pease, 2001]. This ontology is used to define the units of measurement, such as meters and miles in the Length Measurement class (Fig. 5).

\section{Dublin Core ontology}

We followed the Dublin core metadata element set version $1.1^{3}$, to develop the Dublin Core ontology to annotate generic metadata of Gl services, such as title, descriptions, and keywords, with formal meanings.

In the following section, a prototype of $\mathrm{MAKOCl}$ is implemented, and the developed ontologies are used for semantic interoperability of $\mathrm{Gl}$ services.

\section{PROTOTYPE}

We used the following techniques to implement a prototype for the proposed framework. 1) For the ontologies, Protégé software 4 was used as an ontology editor to standardize the domain knowledge. 2) For

\footnotetext{
${ }^{3}$ http://dublincore.org/documents/dces/

${ }^{4}$ http://protege.stanford.edu/
}

the ONTOCAT, the Protégé OWL JAVA API ${ }^{5}$ was used as a programming interface, and the Google Maps JavaScript $\mathrm{API}^{6}$ was used as a base map. 3) For the agents, Java agent developed framework (JADE) ${ }^{7}$ was followed to build a multi-agent system. We used two aspects (i.e. how to register $\mathrm{Gl}$ services and how to search Gl services) to demonstrate the prototype and illustrate the processes in $\mathrm{MAKOCI}$.

\section{Registering $\mathrm{Gl}$ services in $\mathrm{MAKOCl}$}

Web service providers can publish Gl services, including data accessing and geoprocessing services, in the ONTOCAT. The formal meanings in ontologies can be given to the published Gl services for semantic management and discovery by Web service consumers. Three steps are designed for the providers to publish their $\mathrm{Gl}$ services.

First, the providers submit the metadata of the services (e.g., title, URL, coordinate systems, or geometry) into ONTOCAT, as shown in Fig. 6. While submitting, formal knowledge (e.g., classes, individuals, and properties defined in ontologies) can be selected to annotate semantics of the metadata via the user interface, such as dropdown lists to ensure that correct selection of individuals and classes from ontologies. For example, the values in the coordinate system and geometry drop-down lists are from the individuals of Spatial Reference class and Geometry class in the GIS ontology. The hasSpatialReference and hasGeometry properties will be used to annotate the relationship between the $\mathrm{Gl}$ service and selected values.

Nevertheless, for geoprocessing services, the inputs and outputs of geoprocessing services are critical to be annotated with formal meanings in ontologies. ONTOCAT also provides several drop-down lists for providers to select corresponding values from the ontologies, as shown in Fig. 7. For instance, in_buffer is an input parameter of

\footnotetext{
http://protege.stanford.edu/plugins/owl/api/

${ }^{6}$ http://code.google.com/apis/maps/

http://jade.tilab.com/
} 


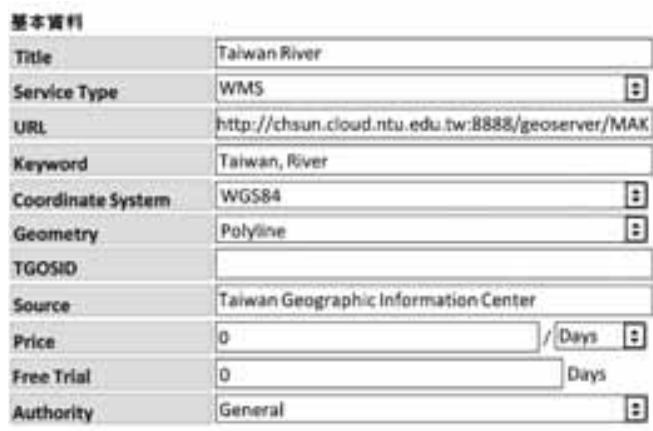

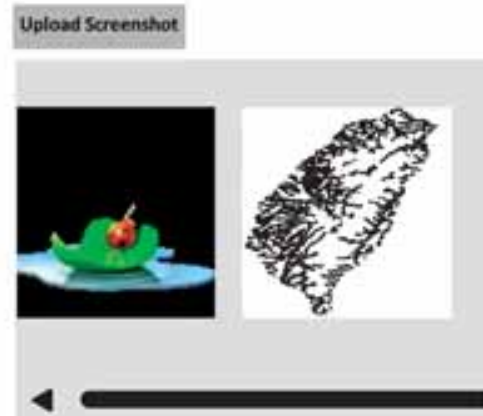

Description

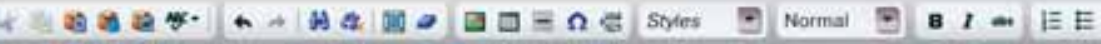
$2 \mathrm{As} \mathrm{P}$

Taiwan River Map

Fig. 6. Screenshots of register data accessing service in ONTOCAT

ws_buffer which have to be implemented

in the WFS standard with the Point geometry and the latitude and longitude

\begin{tabular}{|c|c|c|}
\hline Parameter & Add Prameter & \\
\hline Direction & Input $\div$ & \\
\hline Name & in_buffer & \\
\hline Type & FeatureCollection & $\div$ \\
\hline Web Service Type & WFS : & \\
\hline Geometry & Point & \\
\hline Coordinates & MGS84 $\div$ & \\
\hline Direction & Input $\div$ & \\
\hline Name & distance & \\
\hline Type & xsd:Double & $\div$ \\
\hline Direction & Output $\div$ & \\
\hline Name & out_buffer & \\
\hline Type & FeatureCollection & $\div$ \\
\hline Web Service Type & WFS & \\
\hline Geometry & Polygon $:$ & \\
\hline Coordinates & WGS84 & \\
\hline
\end{tabular}

Fig. 7. A screenshot of annotating the inputs (i.e., in_buffer and distance) and an output (i.e., out_buffer) of ws_buffer geoprocessing service in ONTOCAT coordinate system (i.e., WGS84). Moreover, these metadata of the parameter will be described by corresponding properties, such as hasWebServiceType, hasGeometry, and has SpatialReference, in the ontologies.

Second, providers have to select at least one class from two ontologies, GIS Data Theme ontology for data accessing services and GIS Function Theme ontology for geoprocessing services, to classify GI services. Classes in these

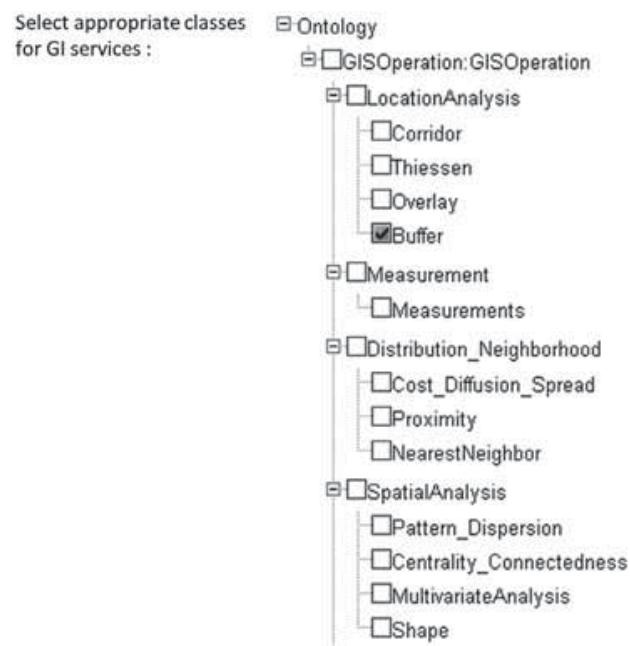

Fig. 8. Select appropriate classes from GIS Function Theme ontology for classifying a geoprocessing service, ws_buffer 


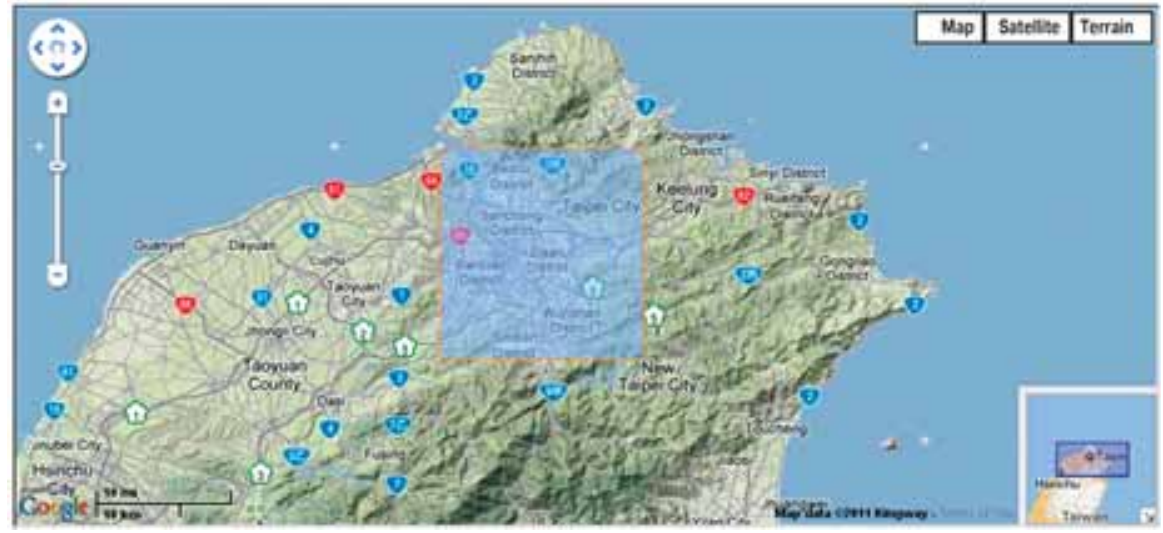

Fig. 9. A rectangular area in Taipei city, Taiwan, is given to express the geospatial extent of ws_buffer

ontologies will be listed in the ONTOCAT for selection, as shown in Fig. 8. For example, Flood class in the GIS Data Theme ontology can be selected for Taiwan River, a data accessing service; or Buffer class in GIS Function Theme ontology can be also chosen for ws_buffer, a geoprocessing service.

Third, a geospatial extent can be given for Gl services to express the geographical area which Gl services can be served. Providers can delimit a rectangular area in ONTOCAT as a service area for a Gl service, as shown in Fig. 9.

Once registered, the metadata of the Gl service will be encoded in RDF, as shown in Fig. 10 for Taiwan_River and Fig. 11 for ws_buffer. The registered metadata of $\mathrm{Gl}$ services will be semantically formalized and presented in RDF triplets so that machines can infer more external resources via these triplets.

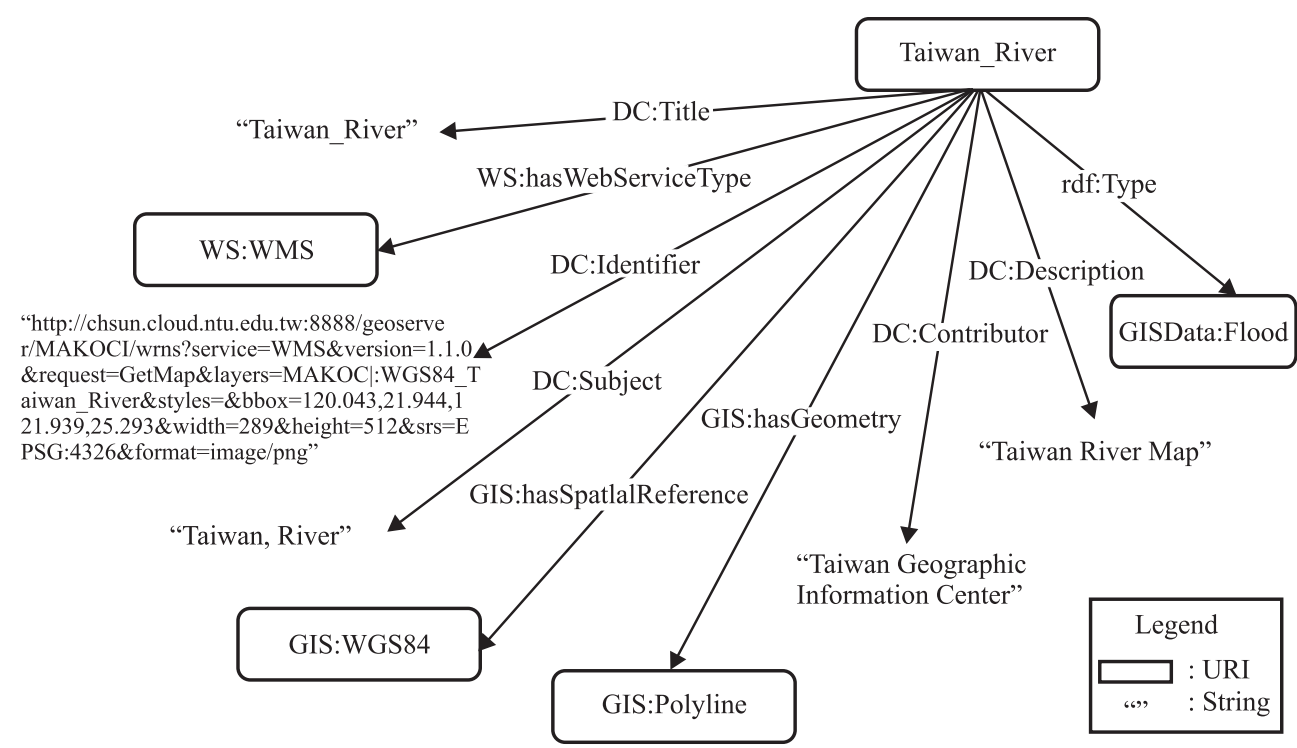

Fig. 10. The registered metadata of Taiwan_River is encoded in RDF, where DC, WS, GIS, and GISData are namespaces of corresponding ontologies, such as Dublin Core, Web Service, GIS, and GIS Data Theme ontologies, respectively 
to integrate and share all these tremendous volume of $\mathrm{Gl}$ services is a major challenge ahead of us. This study presents an initial research effort (i.e., MAKOCl) to this challenge. MAKOCI facilitates ontologies and multiagents to develop a platform for integrating and sharing $\mathrm{Gl}$ services. Ontologies are used for knowledge management and semantic interoperability of Gl services, whereas multi-agents are adopted for intelligently assistances to search and query the Gl services and ontologies. By $\mathrm{MAKOCl}$, users do not need to consider the semantic heterogeneous in each Gl service, but they can use formal meanings of concepts in ontologies to register, manage, and search Gl services. For earth observations, $\mathrm{MAKOCl}$ could be a solution for sharing and integrating Gl services.

However, there are several areas of MAKOCI that need to be improved in the future, as shown in the following issues.

\section{More comprehensive ontologies}

This study designs only six ontologies (as shown in Fig. 4) to describe formal meanings for $\mathrm{Gl}$ services, and only use main classification to build hierarchical relations of classes in the ontologies, such as the 34 categories of INSPIRE are used for the GIS Data Theme ontology. More comprehensive ontologies should be developed in the future to describe completely concepts and relationships for $\mathrm{Gl}$ services

\section{Friendly ontologies editor}

In the prototype, the concepts, individuals, and relationships of ontologies are encoded by using Protégé software. Nevertheless, using Protégé to encode the knowledge is a challenge for many domain experts. Therefore, a Web and Wiki-styled ontology editor would be a significant enhancement, allowing domain experts to directly encode and share domain knowledge in ontologies [lorio et al., 2006].

\section{Connection with Linked Open Data}

Linked Open Data aims to publish machinereadable data on the Web (i.e., RDF) to make the data inter-linkable to other external data sets [Bizer et al., 2009]. Several websites, such as Wikipedia and GeoNames, have joined into the Linked Open Data and converted their data into the RDF format and linked to external relevant data sets to make the meanings of the data explicitly defined. MAKOCI should join into the Linked Open Data and act as a Gl service one-stop for external data sets to establish links. In addition, ONTOCAT should be expanded for Web service providers to input links from external data sets to make all relevant information of $\mathrm{Gl}$ services can be interlinked.

\section{REFERENCES}

1. Albrecht, J. (1998) Universal Analytical GIS Operations - A task-oriented systematisation of data-structure-independent GIS functionality. In Geographic Information Research: transatlantic perspectives, eds. M. Craglia and H. Onsrud, 577-591. London: Taylor \& Francis.

2. Babitski, G., Bergweiler, S., Hoffmann, J., Schön, D., Stasch, C., and Walkowski, A. (2009) Ontology-based integration of sensor web services in disaster management. Paper read at GeoSpatial Semantics 2009, LNCS 5892, at Berlin.

3. Benjamins, V.R., Fensel, D., and Perez, A.G. (1998) Knowledge management through ontologies. Paper read at Proceedings of the Second Inernational Conference on Practical Aspects of Knowledge Management, at Basel, Switzerland.

4. Bernard, L., Einspanier, U., Haubrock, S., Hubner, S., Kuhn, W., Lessing, R., Lutz, M., and Visser, U. (2003) Ontologies for intelligent search and semantic translation in spatial data infrastructures. Photogrammetric-Fernerkundung-Geolnformation (PFG), N. 6, pp. 451-462. 
5. Bishr, Y. (1998) Overcoming the semantic and other barriers to GIS interoperbility. International Journal of Geographic Information Science, N. 12, V. 4, pp. 299-314.

6. Bizer, C., Heath, T., and Berners-Lee, T. (2009) Linked data-the story so far. Int. J. Semantic Web Inf. Syst., N. 5, V. 3, pp. 1-22.

7. Crubézy, M., and Musen, M. A. (2003) Ontologies in support of problem solving. In Handbook on ontologies, eds. S. Staab and R. Studer, 321-341. Berlin: Springer.

8. Di, L., Zhao, P., Yang, W., and Yue, P. (2006) Ontology-driven Automatic Geospatial-Processing Modeling based on Web-service Chaining, Proceedings of the Sixth Annual NASA Earth Science Technology Conference, College Park, MD.

9. Egenhofer, M. (2002) Towards the Semantic Geospatial Web. Paper read at Proceedings of the Tenth ACM International Symposium on Advances in Geographic Information Systems, at McLean, Virginia.

10. Fonseca, F., and Sheth, A. (2002) The Geospatial Semantic Web. Washington, D.C.: University Consortium of Geographic Information Science White Paper.

11. Goodwin, C., and Russomanno, D.J. (2006) An ontology-based sensor network prototype environment. Paper read at Proceedings of the 5th International Conference on Information Processing in Sensor Networks, at Nashville, TN.

12. Gruber, T.R. (1993) A translation approach to portable ontology specifications. Knowledge Acquisition, N. 5, V. 2, pp. 199-220.

13. Hadzic, M., Wongthongtham, P., Dilon, T., and Chang, E. (2009) Ontology-Based MultiAgent Systems. Berlin Heidelberg: Springer.

14. Harvey, F., Kuhn, W., Pundt, H., Bishr, Y., and Riedemann, C. (1999) Semantic interoperability: a central issue for sharing geographic information. The annals of regional science, $\mathrm{N}$. 33, pp. 213-232.

15. Iorio, A. D., Presutti, V., and Vitali, F. (2005) WikiFactory: An Ontology-Based Application for Creating Domain-Oriented Wikis. Paper read at The 3rd European Semantic Web Conference, ESWC 2006, at Budva, Montenegro.

16. Jaeger, E., Altintas, I., Zhang, J., Ludäscher, B., Pennington, D., and Michener, W. (2005) A scientific workflow approach to distributed geospatial data processing using web services. Paper read at SSDBM'2005 Proceedings of the 17th international conference on Scientific and statistical database management, at Santa Barbara, CA.

17. Janowicz, K., Bröring, A., Stasch, C., and Everding, T. (2010) Towards Meaningful URIs for Linked Sensor Data. Paper read at Proceedings of the Workshop "Towards Digital Earth: Search, Discover and Share Geospatial Data 2010" at Future Internet Symposium 2010, at Berlin, Germany.

18. Janowicz, K., Bröring, A., Stasch, C., Schade, S., Everding, T., and Llaves, A. (2011) A RESTful Proxy and Data Model for Linked Sensor Data. International Journal of Digital Earth, N., pp. Manuscript accepted for publication. 
19. Klyne, G., and Carroll, J. J. (2004) Resource description framework (RDF): Concepts and Abstract Syntax, ed. B. McBride.

20. Knublauch, H., Rector, A., Stevens, R., and Wroe, C. (2004) A practical guide to building OWL ontologies using the Protege-OWL plugin and CO-ODE tools edition 1.0, Workshop on OWL: Experiences and Directions, Fourth International Semantic Web Conference (ISWC2005), Galway, Ireland.

21. Kolas, D., Hebeler, J., and Dean, M. (2005) Geospatial Semantic Web: Architecture of Ontologies. Paper read at GeoS 2005, LNCS 3799, at Mexico City, Mexico.

22. Kuhn, W. (2003) Semantic reference systems. International Journal of Geographic Information Science, N. 17, V. 5, pp. 405-409.

23. Kvaløy, T. A., Rongen, E., Tirado-Ramos, A., and Sloot, P. (2005) Automatic composition and selection of semantic web services. Paper read at Advances in Grid Computing-EGC 2005, LNCS 3470, at Berlin, Germany.

24. Lemmens, R. (2006) Semantic interoperability of distributed geo-services, International Institute for Geo-Information Science and Earth Observation (ITC), Delft University of Technology, Enschede, The Netherlands.

25. Lutz, M. (2005) Overcoming Differences of Meaning in Spatial Data Infrastructures Achievement and Challenges, Position Paper for Workshop on Cross-learning between Spatial Data Infrastructures and Information Infrastructures, Enschede, the Netherlands.

26. Lutz, M. (2006) Ontology-based discovery and composition of geographic information services, Institute for Geoinformatics, University of Münster, Münster.

27. Lutz, M., Lucchi, R., Friis-Christensen, A., and Ostländer, N. (2007) A Rule-Based Description Framework for the Composition of Geographic Information Services. In GeoSpatial Semantics, eds. F. T. Fonseca, M. A. Rodriguez and S. Levashkin, 114-127: Springer.

28. Martin, D., Burstein, M., Hobbs, J., Lassia, O., McDermott, D., Mcllraith, S., Narayanan, S., Paolucci, M., Parsia, B., Payne, T., Sirin, E., Srinivasan, N., and Sycara, K. (2004) OWL-S: Semantic Markup for Web Services. W3C 2004 [cited. Available from http://www.w3.org/Submission/OWL-S.

29. McGuinness, D. L., and Van Harmelen, F. (2004) OWL Web Ontology Language overview 2004 [cited 7 June 2012]. Available from http://www.w3.org/TR/owl-features/.

30. Moodley, D., and Kinyua, J. (2006) Towards a multi-agent infrastructure for distributed Internet applications, 8th Annual Conference on WWW Applications, Bloemfontein, South Africa.

31. Moodley, D., and Simonis, I. (2006) A new architecture for the sensor web: the SWAP framework, The 5th International Semantic Web Conference (IWSC), Athens.

32. Niles, I., and Pease, A. (2001) Towards a Standard Upper Ontology. Paper read at the 2nd International Conference on Formal Ontology in Information Systems (FOIS-2001), at Ogunquit, Maine. 
33. OGC. (2005) Web Feature Service Implementation Specification: Technical report OGC 04-094.

34. OGC. (2006a) OpenGIS Implementation Specification for Geographic information - Simple feature access - Part 1: Common architecture Techincal report 06-103r4.

35. OGC. (2006b) OpenGIS ${ }^{\otimes}$ Web Map Server Implementation Specification, version 1.3.0. In Technical report OGC 06-042, ed. O. G. Consortium.

36. Peachavanish, R., and Karimi, H. (2007) Ontological Engineering for Interpreting Geospatial Queries. Transactions in GIS, N. 11, V. 1, pp. 115-130.

37. Peng, Z., and Tsou, M. (2003) Internet GIS: Distributed Geographic Inofrmation Services for the Internet and Wireless Networks. New Jersey: John Wiley \& Sons.

38. Prud'Hommeaux, E., and Seaborne, A. (2008) SPARQL query language for RDF. In W3C working draft.

39. Purves, R.S., Clough, P., Jones, C.B., Arampatzis, A., Bucher, B., finch, D., Fu, G., Joho, H., Syed, A.K., Vaid, S., and Yang, B. (2007) The design and implementation of SPIRIT: a spatially aware search engine for information retrieval on the internet. International Journal of Geographic Information Science, N. 21, V. 7, pp. 717-745.

40. Rauble, M. (2001) Ontology and epistemology for agent-based wayfinding simulation. International Journal of Geographic Information Science, N. 15, pp. 653-667.

41. Sengupta, R., and Sieber, R. (2007) Geospatial Agents, Agents Everywhere... Transactions in GIS, N. 11, V. 4, pp. 483-506.

42. Stock, K. (2008) Determining Semantic Similarity of Behaviour Using Natural Semantic Metalanguage to Match User Objectives to Available Web Services. Transactions in GIS, N. 12, pp. 733-755.

43. Uschold, M. (1998) Knowledge level modeling: concepts and terminology. The Knowledge Engineering Review, N. 13, V. 1, pp. 5-29.

44. Wooldridge, M. (1999) Intelligent Agents. Cambridge, MA: MIT Press.

45. Wooldridge, M., and Jennings, N. R. (1995) Intelligent agents: Theory and practice. Knowledge engineering review, N. 10, V. 2, pp. 115-152.

46. Yang, C., Raskin, R., Goodchild, M., and Gahegan, M. (2010) Geospatial Cyberinfrastructure: Past, present and future. Computers, Environment and Urban Systems, N. 34, pp. 264-277.

47. Yu, L. (2011) A Developer's Guide to the Semantic Web. Berlin: Springer.

48. Zhang, T., and Tsou, M. H. (2009) Developing a grid-enabled spatial Web portal for Internet GIServices and geospatial cyberinfrastructure. International Journal of Geographical Information Science, N. 23, V. 5, pp. 605-630. 

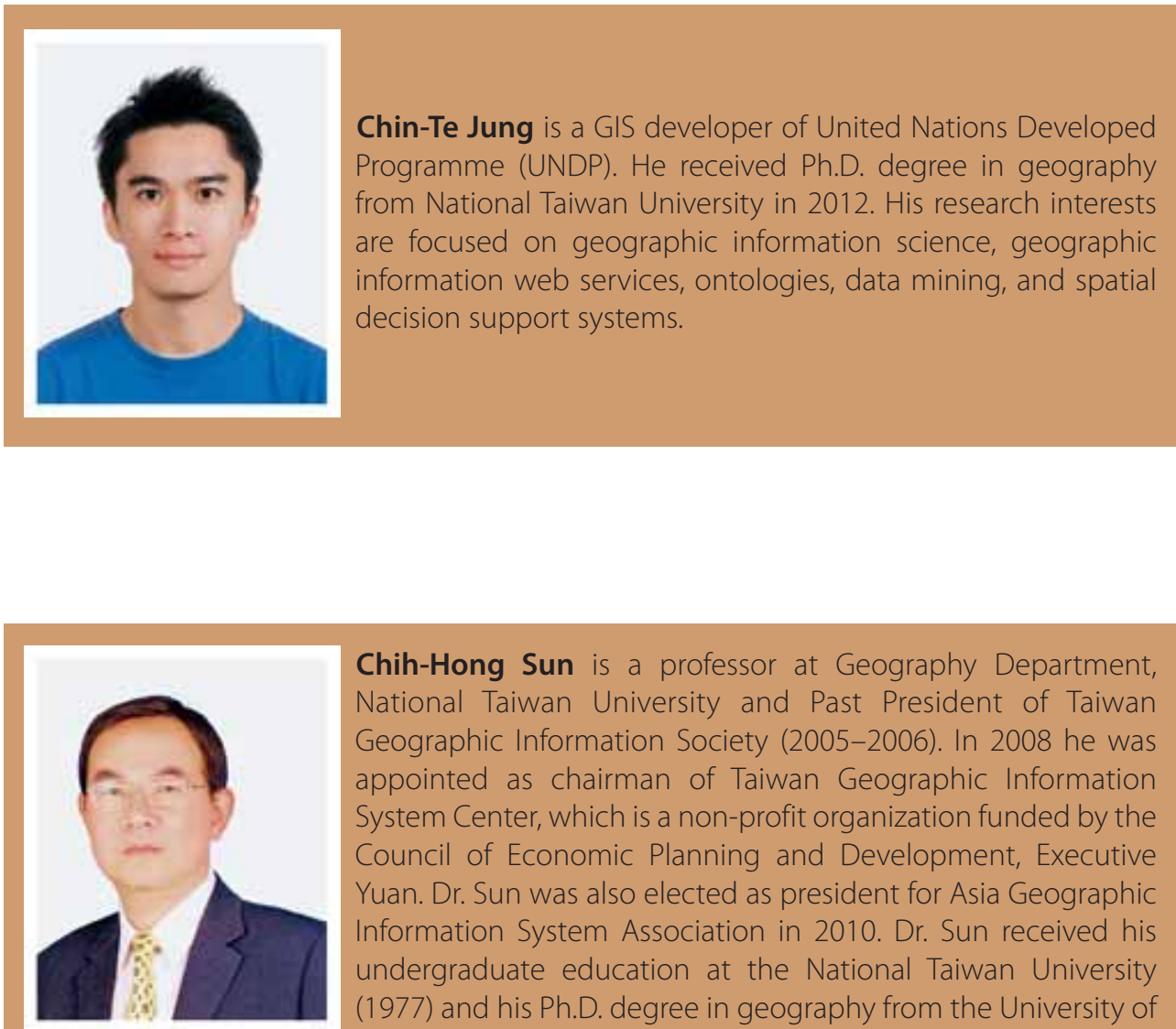

Chih-Hong Sun is a professor at Geography Department, National Taiwan University and Past President of Taiwan Geographic Information Society (2005-2006). In 2008 he was appointed as chairman of Taiwan Geographic Information System Center, which is a non-profit organization funded by the Council of Economic Planning and Development, Executive Yuan. Dr. Sun was also elected as president for Asia Geographic Information System Association in 2010. Dr. Sun received his undergraduate education at the National Taiwan University (1977) and his Ph.D. degree in geography from the University of Georgia, USA (1986). He served as director of the Global Change Research Center, National Taiwan University from 1998 to 2004 and the executive secretary of the Commission on Sustainable Development Research, National Science Council from March, 1998 to June, 2000. Prior to that, Dr. Sun also served as chairman of the geography department, National Taiwan University from August 1994 till July 1997. His research specialties are in geographic information system, decision support system, hazards mitigation, and sustainable development.

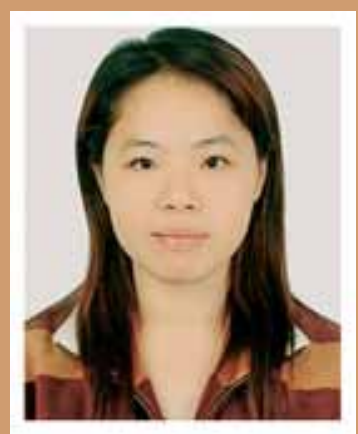

Min-Fang Lien was born in Taiwan. She majored in geography and management. Since graduated from university, she has been working in IT-related companies. Now she is the director of technical division in Taiwan GIS center and also a PhD. student of Department of geography, National Taiwan University. Minfang's specialties are GIS, system analysis and project management. Her recent researches are wireless sensor network, GIS in agriculture. 
Chih-Shyang Chang received the Ph.D. degree in computer science from National Tsing Hua University at Hsinchu, Taiwan, R.O.C. in 1996. He is currently an Assistant Professor in the Department of Information Science at Tungnan University, New Taipei City, Taiwan, R.O.C.. His research interests include data mining, software engineering and web mining.

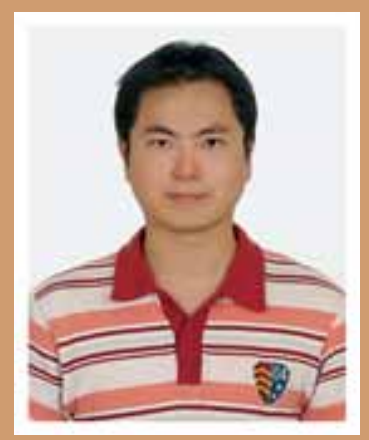

Wei-Jen Chung obtained his Master's degree of Computer Science in 2004. The main idea of his master's dissertation is how to combine ontology with a multi-agent system by Web services, which were part of semantic web architecture. The focus of his research lies on information technology (JADE RDF). He also loves to learn something new. His wish is to establish some systems that can make the world different, and he is still working on it.

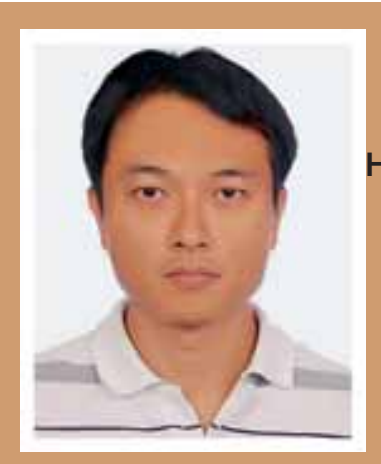

Hong-Yang Lin studied Electronic and Computer Engineering at National Taiwan University of Science and Technology and graduated in 2000. Since June 2010 he is a system engineer of the Taiwan GIS Center.

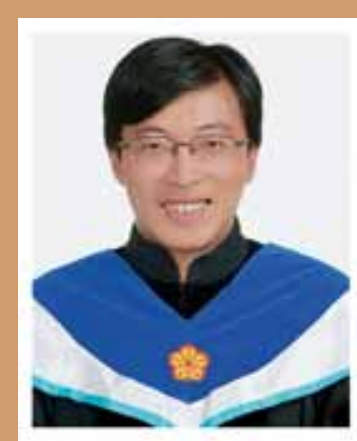

Ping-Ying Tsai is an engineer of Taiwan Geographic Information System Center. He received Ph.D. degree in computer science from National Taiwan University in 2008. He served as an assistant professor at Department of Computer Science and Information Engineering, Hwa Hsia Institute of Technology from September 2008 to July 2009. After that, Dr. Tsai served as a postdoctoral fellow at Institute of Mathematics, Academia Sinica from August 2009 to July 2011. His research specialties are graph theory, discrete mathematics, algorithms, and interconnection networks. 